\title{
Applicability of Wireless Activity Sensor Network to Avian Influenza Monitoring System in Poultry Farms
}

\author{
Hironao Okada1, Koutarou Suzuki², Tsukamoto Kenji², Toshihiro Itoh' ${ }^{1}$ \\ ${ }^{1}$ National Institute of Advanced Industrial Science and Technology, Tsukuba, Japan \\ ${ }^{2}$ National Institute of Animal Health, Tsukuba, Japan \\ Email: hironao.okada@aist.go.jp
}

Received 27 January 2014; revised 24 February 2014; accepted 3 March 2014

Copyright (C) 2014 by authors and Scientific Research Publishing Inc.

This work is licensed under the Creative Commons Attribution International License (CC BY). http://creativecommons.org/licenses/by/4.0/

(c) (i) Open Access

\begin{abstract}
Since there are cases that chickens infected with highly pathogenic avian influenza (HPAI) viruses die with almost no fever, body-temperature sensing cannot be effective for the early detection of avian influenza (AI) infection in these cases. In addition, sensors directly attached to the body surface are easily affected by their surroundings, therefore it is not easy to measure the body-temperature of chickens. This paper proposes a new method to detect abnormal states of chickens with only their activity data obtained by wearable wireless sensor nodes. The method is that if its state that the present average activity of a chicken in one hour is smaller than 0.6 times minimum average daytime activity in its life continues for 3 hours, a judgment that the chicken is abnormal state is made. This method can detect the abnormal states twice as early as that with body-temperature sensing does. Since the activity sensor nodes are easily attached to chickens and hardly affected by their surroundings, this system can be reliable.
\end{abstract}

Keywords

Activity Sensor; Wireless Sensor Network; Avian Influenza Surveillance System

\section{Introduction}

Wireless sensor networks that consist of a large number of wireless sensor nodes attached to, or embedded into, animal and human body or other objects can be applied to monitoring systems of their conditions and related ambient data for realizing green [1], safe [2] and secure [3] society. As an application of wireless sensor network, our group has been developing a global avian influenza (AI) surveillance system by monitoring the health of 
chickens with wireless sensor nodes in poultry farms [4] [5]. As is well known, the highly pathogenic avian influenza (HPAI) virus (H5N1) infection in birds has continued during these years [6], and has acquired pathogenicity not only in birds but also in mammals [7]. The more cases of migratory birds and domestic fowls increase, the more human cases increase and the variation of the virus progresses. Consequently, risks of occurrence of a pandemic flu with transmissibility among humans increase. Therefore a global avian influenza surveillance system for the early-stage detection of birds cases must be effective to maintain public health by defending human beings from an influenza pandemic.

In Japan, the aging and decrease of the workers in the family-scale poultry farms are seriously advancing, and on the other hand, the poultry farms have been scaling up rapidly in recent years. Thus signs of abnormality of chickens' health conditions that could previously be detected by the worker scan are easily missed. In addition, it is difficult for the conventional patrol to detect the sings due to heat stress in summer, for instance. It is hence desirable to introduce a technology that allows automatic health monitoring of the chicken population in the farms, for not only the early detection of avian influenza outbreak, but also the improvement of productivity. Compared to the current patrol-based detection system in which the workers can recognize the abnormality when 3 to 5 chickens in a cage die at the same time, the automatic sensing system may find the abnormality even when only two neighbor chickens have a health condition change with a time difference.

Accordingly, we have been developing wireless body-temperature and activity sensor nodes that can detect the individual chicken health condition [5]. Due to AI infection experiments using the prototype wireless senor nodes [8] [9], it was found that body-temperature profile sensing can be effective to obtain the information related to the pathogenicity of virus; on the other hand, it was noticed that there are cases that chickens infected with HPAI viruses die without the distinct body-temperature rise [8]. In addition, it is not easy to attach the temperature sensor probe directly to the body surface and to keep the long-term reliability because the contact condition between the probe and body can be easily changed due to the movement and its sensing data can be affected by its surroundings.

In this study, it is examined the possibility of applying the wireless nodes with only an activity sensor to the HPAI infection monitoring of chickens. Activity sensing is hardly affected by the attachment condition and the surroundings, and activity sensor itself can be provided with a very low cost due to recent diffusion of micro-fabricated accelerometers in wearable information devices such as smart phones. However, in the previous studies [5], it has not been discussed if the activity sensing alone is applicable to the early-detection of AI infection possibility. If the number of sensors in the node can be minimized into only one, that contributes to not only the reduction of sensor node production cost, but also the communication traffic; it will help the sensor nodes to work longer or have smaller battery as well.

\section{Activity Sensing of AI Infected Chickens}

Figure 1 illustrates the prototype wireless sensor node that was utilized for AI infection experiments of chickens. The H34C (HITACHI Metals Ltd.) of 3-axis accelerometer with current consumption of $1 \mu$ A during the sleep state is equipped as an activity sensor. The 3-axis acceleration is recorded at 20 times per one measurement which takes about 0.14 milliseconds. By eliminating the gravity, the activity $(A)$ is calculated as:

$$
\begin{gathered}
A_{C x}=\sum_{n=1}^{20}\left|A x_{n}-A x_{n-1}\right|, A_{C y}=\sum_{n=1}^{20}\left|A y_{n}-A y_{n-1}\right|, A_{C z}=\sum_{n=1}^{20}\left|A z_{n}-A z_{n-1}\right| \\
A=\sqrt{A c_{x}^{2}+A c_{y}^{2}+A c_{x z}^{2}}
\end{gathered}
$$

where $A x_{n}, A y_{n}$ and $A z_{n}$ are 3-axis accelerations. $A_{c x}, A_{c y}$, and $A_{c z}$ are sent to the receiver from the sensor nodes. A micro controller unit (MCU) and a transceiver IC for the industry-science-medical (ISM) band at 2.4 - 2.525 $\mathrm{GHz}$ are the C8051F411 (Silicon Laboratories) [10] with 12 bit analog-to-digital converter (ADC) and the nRF24L01 (Nordic Semiconductor) [11] with $900 \mathrm{nA}$ current consumption in power down mode, respectively. The modulation method is Gaussian filtered frequency shift keying (GFSK), and the communication distance was approximately $20 \mathrm{~m}$ without obstacles. A radial lead thermistor is also mounted on the nodes as a bodytemperature sensing probe. Then the total size and weight are $\varphi 24 \mathrm{~mm} \times \mathrm{t} 9 \mathrm{~mm}$ and $5.2 \mathrm{~g}$, respectively, including the battery (CR1632, $1.7 \mathrm{~g}$ ) and a plastic cover case (2.2 g). The sensor nodes can continuously work in 2 weeks and longer, by adopting uni-directional system and 20 seconds intermittent operation. 


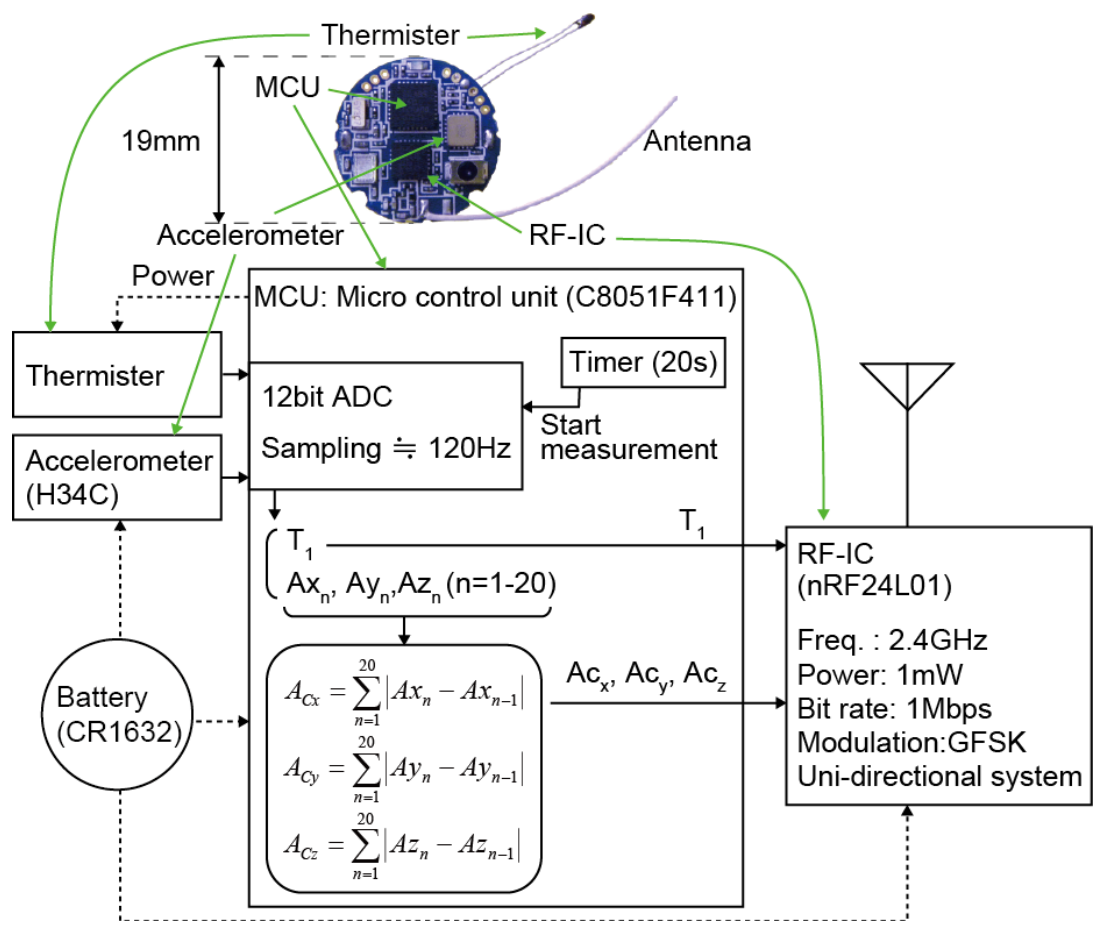

Figure 1. Photograph and block diagram of the prototype wireless sensor node.

In the AI infection experiments, the sensor node was attached to the abdominal skin surface of a chicken with non-stretching sport tape. Four-week-old specific-pathogen-free (SPF) chickens were used for the experiments. The wireless signals from the sensor nodes were detected a universal serial bus (USB) type receiver placed outside of experimental isolators. In this study, we used the data of 10 chickens infected with the influenza virus, $\mathrm{A} /$ chicken/Miyazaki/K11/2007(H5N1); CkMZ11, obtained in the transmission experiment [9]. The CkMZ11 is the virus that most recently did the greatest damage to the poultry industry in Japan.

Table 1 shows basic data for 10 infected chickens obtained in the experiment of infection transmission [9]. Since only the data of indirectly-infected chickens (without those of directly-inoculated chickens) are used, the estimated infection and death times vary with the chickens. The experimental data indicates the sensed bodytemperature of chickens was from $39^{\circ} \mathrm{C}$ to $41^{\circ} \mathrm{C}$ in health. Times of death and infection detection are determined by the criteria that the sensed body-temperature is lower than $30^{\circ} \mathrm{C}$, and over $42^{\circ} \mathrm{C}$ or below $38^{\circ} \mathrm{C}$, respectively. The average detection time which is time difference between death and detection with body-temperature sensor is 7.6 hours.

Figure 2 shows examples of activities (blue dots) and body-temperatures (red line) data of chickens infected with CkMZ11. Diurnal activity rhythm that a chicken sleeps for about 8 hours and is active for the rest of the dayis shown. According to the experimental study concerning the body-temperature sensing [8], it was found that fever of chickens infected with a super strong virus, A/chicken/Yamaguchi/7/2004(H5N1); CkYM7 was almost not observed. Comparison of Figures 2(a) and (b) indicates that even in the CkMZK11 cases, elevation of body-temperature due to the infection is sometimes clear, but sometimes not. However it was found that activity decreased and diurnal activity rhythm was broken due to the progress of influenza symptom in both cases. For example in Figure 2(a), although the activity should be increased at 158 hif the chicken was healthy, low activity state continued. Next, we examined concretely how fast the abnormal states can be detected with only activity data.

\section{Infection Possibility Detection Method}

Figures 2(a) and (b) clearly indicate that the activity of chickens decreases with progress of the influenza symptom. Although the detection can be realized with an image processing method for the graphs, accuracy may be decreased. Because this graphs are secondary source made from original activity data obtained from a sensor. Thus, we examined a method in which original activity data was directly utilized. 
Table 1. Basic data for 10 infected chickens obtained in the transmission infection experiment [9].

\begin{tabular}{ccccc}
\hline ID & *Estimated infected time (h) & Time of death (h) & $\begin{array}{c}\text { Time of detection with body-temp. } \\
\text { sensor (h) }\end{array}$ & $\begin{array}{c}\text { Time difference between death and } \\
\text { detection (detected health state) (h) }\end{array}$ \\
\hline 1 & 60 & 111 & 109 & 2 (Low body-temp.) \\
2 & 62 & 113 & 110 & 3 (Low body-temp.) \\
3 & 66 & 117 & 114 & 3 (Low body-temp.) \\
4 & 81 & 132 & 113 & 19 (Fever) \\
5 & 64 & 115 & 111 & (Low body-temp.) \\
6 & 113 & 164 & 155 & 9 (Fever) \\
7 & 117 & 168 & 166 & 2 (Low body-temp.) \\
8 & 122 & 173 & 159 & 2 (Fever) \\
9 & 130 & 181 & 179 & 18 (Fever) \\
10 & 131 & 182 & 164 & 7.6 \\
\hline
\end{tabular}

${ }^{*}$ Estimated infected time $=$ Time of death-Mean death time (MDT). MDT is $51.4 \mathrm{~h}$ [9].
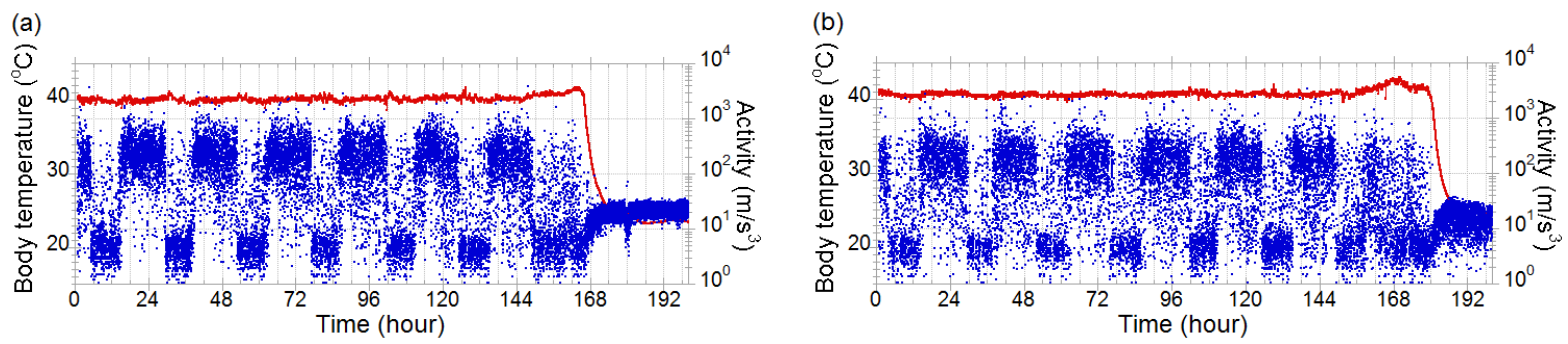

Figure 2. Examples of activities (blue dots) and body-temperatures (red line) data of chickens infected with CkMZ11. (a) and (b) are the cases that fever is notclearly shown and is clearly seen, respectively. The ID numbers of (a) and (b) in Table 1 are 7 and 10, respectively.

Here we use one hour average of activity $A 1 h_{t}$ which can be calculated by the simple average of $A$ in an hour. The subscription of $A 1 h_{t}$ is time. Previously, we compared $A 1 h_{t}$ with $A 1 h_{t-24}$ which is 1 hour average of activity before 24 hours [5] and if the difference of $A 1 h_{t}$ with $A 1 h_{t-24}$ exceeds a threshold, we made a judgment that the chicken is abnormal state. In the case of that $A 1 h_{t-24}$ becomes small by accident, if $A 1 h_{t}$ is small caused by HPAI infection, the abnormal state could not be detected because of the small activity difference. The average detection time was about 6 hours by this method and detection ratio that abnormal states can be detected earlier than the deaths with only activity data is not $100 \%$.

In this study, minimum average of daytime activities $A d_{\text {ave-min }}$ when chickens had not been infected (before the infected time shown in Table 1 ) is used instead of $A 1 h_{t-24}$. To calculate the average activity, we must discriminate between daytime and night time of each chicken. From the experimental data summarized in Table 1, it is settled that the wake-up and bed times are determined by

$$
\begin{aligned}
& \text { Wake-up time: } A 1 h_{t-1}<150\left(\mathrm{~m} / \mathrm{s}^{3}\right) \cap A 1 h_{t}>150\left(\mathrm{~m} / \mathrm{s}^{3}\right) \\
& \text { Bed time: } \quad A 1 h_{t-1}>100\left(\mathrm{~m} / \mathrm{s}^{3}\right) \cap A 1 h_{t}<100\left(\mathrm{~m} / \mathrm{s}^{3}\right)
\end{aligned}
$$

respectively, and the average of daytime activities $A d_{\text {ave }}$ is calculated by averaging $A 1 h_{t}$ from 2 hours after wake-up time to 2 hours before bedtime in the previous day. It is supposed that daytime starts from the previous day's wake-up time and ends at the previous day's bed time. Since $A 1 h$ varies widely in daytime, it is concluded that the chicken has AI infection possibility when the activities during the latest 3 hours are lower than the minimum $A d_{\text {ave-min, }}$

$$
A 1 h_{t}<\alpha A d_{\text {ave-min }} \cap A 1 h_{t-1}<\alpha A d_{\text {ave-min }} \cap A 1 h_{t-2}<\alpha A d_{\text {ave-min }}
$$

where $\alpha$ is determined from the experimental data.

Figures 3(a) and (b) show the 1 hour average activity (blue dotted line) and average of daytime activities 

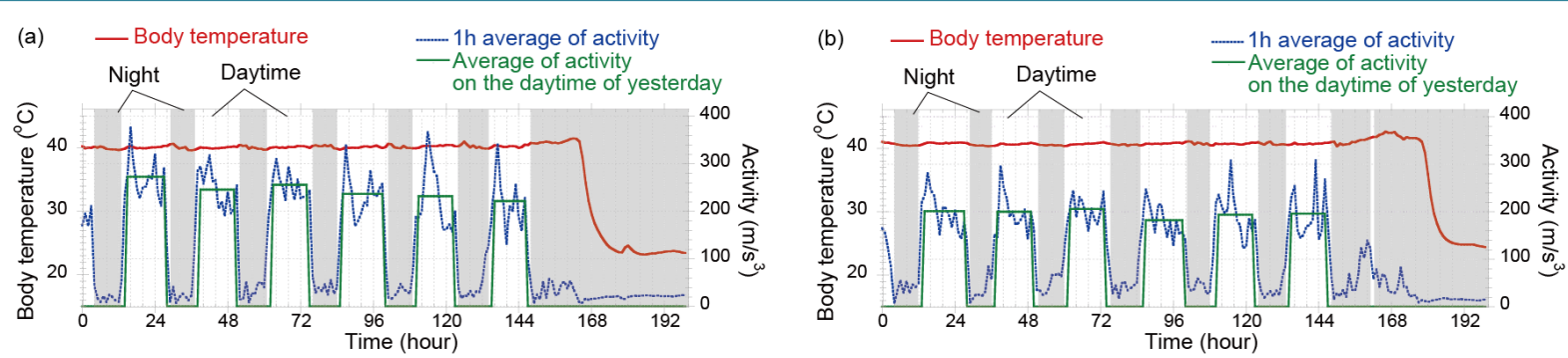

Figure 3. (a) and (b) show the 1 hour average activity (blue line) and average of daytime activities (green line), derived from the data shown in Figures 2(a) and (b), respectively.

Table 2. $\alpha$ dependence of the time difference between death and infection possibility detection with the maximum standard deviation of daytime activities for each chicken.

\begin{tabular}{|c|c|c|c|c|c|c|c|}
\hline \multirow{3}{*}{ ID } & \multicolumn{6}{|c|}{ Time difference between death and detection (h) } & \multirow{3}{*}{$\begin{array}{c}\text { Maximum standard deviation/average activity during daytime } \\
\text { (\%) }\end{array}$} \\
\hline & \multicolumn{6}{|c|}{$\alpha$} & \\
\hline & 0.5 & 0.55 & 0.6 & 0.65 & 0.7 & 0.75 & \\
\hline 1 & 1 & 1 & 1 & 1 & 1 & 1 & 37.7 \\
\hline 2 & 3 & 17 & 17 & 17 & 19 & 19 & 40.9 \\
\hline 3 & 7 & 7 & 7 & 7 & 19 & 19 & 35.9 \\
\hline 4 & 14 & 34 & 34 & 36 & 37 & 37 & 33.8 \\
\hline 5 & 28 & 28 & 28 & 42 & 45 & 45 & 36.9 \\
\hline 6 & 5 & 5 & 5 & 5 & 5 & 5 & 27.3 \\
\hline 7 & 8 & 8 & 8 & 8 & 8 & $* 47$ & 29.3 \\
\hline 8 & 14 & 14 & 14 & 14 & 14 & 14 & 26.9 \\
\hline 9 & 17 & 17 & 17 & 17 & 20 & 20 & 42.5 \\
\hline 10 & 18 & 18 & 18 & 18 & 23 & 23 & 27.4 \\
\hline Average & 11.5 & 14.9 & 14.9 & 16.5 & 19.1 & 20.3 & 33.9 \\
\hline
\end{tabular}

*False detection.

(green line), derived from the data shown in Figures 2(a) and (b), respectively. And Table 2 summarizes $\alpha$ dependence of the time difference between death and infection possibility detection with the maximum standard deviation of daytime activities for each chicken and indicates that the detection ratio is $100 \%$. For example in the case of Figure 3(a) and 0.5 of $\alpha$, the condition of Equation (5) is met at $160 \mathrm{~h}$. Only in case of $\alpha=0.75$, an infection possibility was detected in error at the time when the chicken still might have no health problems. Compared with the standard deviation of daytime activities and taking the stability of judged time into account, it can be concluded that 0.6 can be an appropriate value for $\alpha$. Although there is only a case that the detection is just an hour before death judgment, the average of time difference between death and detection when using the activity sensing in this study is 14.9 hours. This value is approximately twice of that when using the body-temperature sensing. It means that the activity sensing can detect the infection possibility earlier than the body-temperature sensing in average, and be effective for an AI surveillance sensor network system.

\section{Conclusion}

We demonstrated that this method can detect abnormal states caused by HPAI infection twice as early as that with body-temperature sensing does. Since the activity sensor nodes are easily attached to chickens and hardly affected by their surroundings compared to the body-temperature sensor nodes, the system can be cost-effective and reliable.

\section{Acknowledgements}

This research is granted by the Core Research for Evolutional Science and Technology (CREST) of Japan Science and Technology Agency (JST) and the Japan Society for the Promotion of Science (JSPS) through the 
“Funding Program for World-Leading Innovative R\&D on Science and Technology (FIRST Program)”, initiated by the Council for Science and Technology Policy (CSTP).

\section{References}

[1] Hayes, J., Beirne, S., King-Tong, L. and Diamond, D. (2008) Evaluation of a Low Cost Wireless Chemical Sensor Network for Environmental Monitoring. Proceedings of IEEE SENSORS, Lecce, 26-29 October 2008, 530-533.

[2] Taylor, S.G., Farinholt, K.M., Flynn, E.B., Figueiredo, E., Mascarenas, D.L., Moro, E.A., Park, G., Todd, M.D. and Farrar, C.R. (2009) A Mobile-Agent-Based Wireless Sensing Network for Structural Monitoring Applications. Measurement Science \& Technology, 20, 1-14. http://dx.doi.org/10.1088/0957-0233/20/4/045201

[3] van de Ven, P., Feld, R., Bourke, A., Nelson, J. and Laighin, G.O. (2008) An Integrated Fall and Mobility Sensor and Wireless Health Signs Monitoring System. Proceedings of IEEE SENSORS, Lecce, 26-29 October 2008, 625-628.

[4] Okada, H., Nogami, H., Kobayashi, T., Masuda, T. and Itoh, T. Ultra-low Power MEMS Activity Sensor for Wireless Health Monitoring System. IEEJ Transactions on Sensors and Micromachines, 134, in press.

[5] Okada, H., Suzuki, K., Tsukamoto, K. and Itoh, T. (2009) ireless Sensor System for Detection of Avian Influenza Outbreak Farms at an Early Stage. Proceedings of IEEE Sensors, Christchurch, 25-28 October 2009, 1374-1377.

[6] Tsukamoto, K., Imada, T., Tanimura, N., Okamatsu, M., Mase, M., Mizuhara, T., Swayne, D. and Yamaguchi, S. (2007) Impact of Different husbandry Conditions on Contact and Airborne Transmission of H5N1 Highly Pathogenic Avian Influenza Virus to Chickens. AVIAN Diseases, 51, 129-132. http://dx.doi.org/10.1637/0005-2086(2007)051[0129:IODHCO]2.0.CO;2

[7] WHO, Influenza Situation Updates—Avian Influenza. http://www.who.int/influenza/human animal interface/avian influenza/archive/en/index.html

[8] Suzuki, K., Okada, H., Itoh, T., Tada, T., Mase, M., Nakamura, K., Kubo, M. and Tsukamoto, K. (2009) Association of Increased Pathogenicity of Asian H5N1 Highly Pathogenic Avian Influenza Viruses in Chickens with Highly Efficient Viral Replication Accompanied by Early Destruction of Innate Immune Responses. Journal of Virology, 83, 74757486. http://dx.doi.org/10.1128/JVI.01434-08

[9] Suzuki, K., Okada, H., Itoh, T., Tada, T. and Tsukamoto, K. (2010) Phenotypes Influencing the Transmissibility of Highly Pathogenic Avian Influenza Viruses in Chickens. Journal of General Virology, 91, 2302-2306. http://dx.doi.org/10.1099/vir.0.023267-0

[10] Silicon Laboratories: Datasheets of C8051F93x-C8051F92x, 67.

[11] Nordic Semiconductor: Datasheets of nRF24L01, 14. 